ISSN 1997-5902

\title{
Étude des lésions anatomo-pathologiques des Tilapia et Clarias vendus sur les marchés de Lubumbashi, République Démocratique du Congo
}

\author{
1 Ipungu L, ${ }^{1}$ Ngoy K, ${ }^{2}$ Pilato J J, ${ }^{3}$ Banze K, ${ }^{3}$ Lumfwa K \\ 1 Service de Pisciculture et Pathologie des Poissons. Fac. Méd. Vét. UNILU. B. P. 1825 R. D. Congo \\ 2 Service d'Anatomie Pathologique, d'Histopathologie, de Physiopathologie et d'Autopsie, Fac. Méd. Vét. UNILU. B. \\ P 1825 R. D Congo. ${ }^{3}$ Service de Parasitologie. Fac. Méd. Vet. UNILU. B. P 1825 R.D Congo. \\ Corresponding author email: ipunguraymond@yahoo.fr, marcelngoy@yahoo.fr, ibanze@hotmail.com, \\ klumfwa@yahoo.com
}

Original submitted in on $16^{\text {th }}$ October 2014. Published online at www.m.elewa.org on $29^{\text {th }}$ December 2014 http://dx.doi.org/10.4314/jab.v84i1.6

\begin{abstract}
RESUME
Objectif : Cette étude a pour objectif d'évaluer la qualité du produit de pêche commercialisé et consommé à Lubumbashi.

Méthodologie et résultats : L'examen macroscopique, l'autopsie de 60 Tilapia spp et 40 Clarias spp ainsi que l'analyse statistique ont été utilisés à cette fin.

L'étude des différentes lésions anatomo-pathologiques a montré que:

$1^{\circ}$ Les processus altératifs observés chez les deux catégories des poissons représentent 42,26p.100 et 23,95p.100 respectivement chez Clarias spp et Tilapia spp.

$2^{\circ}$ Les troubles circulatoires représentent 57,29p.100 chez Tilapia spp et 8,24p.100 chez Clarias spp, et les troubles de la croissance cellulaire 12,5p.100 chez Tilapia spp et 10,30p.100 chez Clarias spp.

Beaucoup de lésions anatomo- pathologiques observées chez Tilapia spp et Clarias spp vendus sur les 3 marchés de Lubumbashi témoignent de la mauvaise qualité du produit consommé à cause du danger présenté pour l'homme (toxi- infections).

Conclusion et application des résultats : Ainsi nous recommandons aux autorités de la province du Katanga de procéder à la séquestration des produits de pêche qui présentent un danger pour la population.

Mots- clés: Poisson, Tilapia, Clarias, lésions anatomo-pathologiques, marché, Lubumbashi.
\end{abstract}

\begin{abstract}
Objective: This study is designed to evaluate the quality of the fishing product marketed and consumed in Lubumbashi.

Methodology and results: The macroscopic examination, the autopsy of 60 Tilapia spp. and 40 Clarias spp. and statistical analysis were used for this purpose. The study of different pathological lesions has shown that:

$1^{\circ}$ the alterative process observed in the two categories of fish represent 42, 26p.100 and 23, 95p.100 respectively in Clarias spp. and Tilapia spp.

2. The circulatory disorders represent $57,29 \mathrm{p} .100$ in Tilapia spp and Clarias spp 8,24p.100 home and disorders 12,5p.100 cell growth in Tilapia spp and Clarias spp 10,30p.100 home.
\end{abstract}


Many pathological anatomical lesions observed in Tilapia spp and Clarias spp sold in 3 markets of Lubumbashi reflect the poor quality of the product consumed because of the danger posed to humans (toxic infections). Conclusion and application of results: Thus we recommend that the province of Katanga authorities to sequestration of fishing products that pose a danger to the public

Key-words: Fish, Tilapia, Clarias, pathological lesions, market, Lubumbashi.

\section{INTRODUCTION}

Les Poissons ont été à l'honneur depuis le temps jadis dans la vie de l'homme et constituent encore de nos jours la base de l'alimentation de plusieurs populations (Mboyo, 1993). En R. D Congo, ils sont abondants et de diverses espèces. Ils sont un excellent aliment par leur richesse en protides, calcium, phosphore et en vitamines. Non seulement ils sont, nutritifs, substantiels mais ils sont faciles à digérer. De ce fait, il est souhaitable, comme l'a souligné Kestemont (2013) qu'ils rentrent le plus largement possible dans l'alimentation humaine. Selon De Kinkelin, Christian et Pierre (1985), Kestemont, Falter et Micha (1989), Losson (2002), Philippart (2005), Daube (2013), les produits de la pêche et de l'aquaculture, outre le rôle joué dans l'alimentation, sont parfois à la base de diverses maladies qui dénaturent les caractères

\section{ANIMAUX, MATERIEL ET MÉTHODES}

Animaux: La catégorie, le nombre et l'origine des poissons de Lubumbashi sont présentés dans le tableau 1. Les poissons ont été pêchés :

A Kapolowe dans le lac Changalele; A Lualaba dans le fleuve Congo; A Lubumbashi dans les étangs de Naviundu et de Kipopo.

\section{Méthodes}

Examen macroscopique: II a été opéré pour l'appréciation des lésions externes, leur taille, type, état des branchies, des yeux, de la peau (Clarias) et des écailles(Tilapia).

Autopsie : Elle a permis de rechercher les lésions au niveau du tissu conjonctif sous-cutané, des muscles, des viscères abdominaux et thoraciques, à l'éosine et à l' hématoxyline.

Anémie: L'anémie est l'appauvrissement du sang, caractérisé par la diminution notable d'un, de plusieurs, ou de tous ses éléments (anémie totale, anémies partielles) (Garnier, 2000)

La notion d'anémie distingue :

-l'anémie qui peut être : organoleptiques et prédisposent l'homme à de toxiinfections et des zoonoses.

L'étude des lésions anatomo-pathologiques sur les poissons vendus et livrés à la consommation humaine s'avère très indispensable car beaucoup $d$ ' entre elles sont des indicatrices des pathologies humaines dont l'impact sur la santé humaine est très évident.

Ainsi la présente étude se propose:

- de décrire les différentes lésions anatomopathologiques observées chez Tilapia spp et Clarias spp;

- d'évaluer leur fréquence par catégorie de poisson dans le cadre de la gestion du produit de la Pêche et de l'aquaculture;

- d'établir éventuellement la relation entre les diverses lésions anatomo-pathologiques et les catégories des poissons.

- $\quad$ générale : lorsque la diminution globale de sang est accompagné de la réduction numérique de ses éléments tels que les hématies. C'est une déchéance qualitative du sang. L'anémie générale sans déchéance qualitative du sang est qualifiée d'oligémie.

- locale ou ischémie lorsqu'il s'agit d'une conséquence d'un trouble circulatoire des artères locales. Catarrhe : Le catarrhe est un nom donné par les anciens à toutes les inflammations aiguës ou chroniques des muqueuses avec hypersécrétion de glandes de la région enflammée (Garnier, 2000).

Congestion(Hyperémie) : La congestion est l'excès de sang dans les vaisseaux d'un organe ou d'une partie d'organe (Garnier, 2000).

Endophtalmie : L'éndophtalmie est l'inflammation de tissus internes de l'œil (Garnier, 2000).

Hémorragie : On appelle hémorragie, l'effusion plus ou moins considérable de sang hors d'un vaisseau sanguin (Garnier, 2000).

Hypertrophie : Elle est définie par une augmentation de la taille des cellules, associée à une augmentation de la 

marchés de Lubumbashi, République Démocratique du Congo

taille de l'organe. Ainsi l'organe hypertrophié n'est pas constitué de nouvelles cellules, mais uniquement des cellules plus volumineuses (Cotran, Kumar, Collins, 2000).

Macroscopiquement : -consistance dure et ferme dans l'hypertrophie d'organes fortement vascularisés

-l'hypertrophie du cœur est générale ou partielle (localisée à une ou plusieurs cavités cardiaques. L'hypertrophie du ventricule gauche allonge l'organe, celle du ventricule droit le rend globulaire)

-hypertrophie concentrique: si s'accompagne de la réduction des cavités avec augmentation des parois

-hypertrophie excentrique si elle s'associe à une dilatation du même organe (cavités agrandies+parois épaisses).

Hydrothorax : L'hydrothorax est un épanchement séreux de la cavité pleurale, tantôt unilatéral, tantôt bilatéral, sans réaction inflammatoire, s'accompagnant généralement d'autres œdèmes et survenant au cours de certaines affections cardiaques et rénales (GARNIER, 2000).

Inflammation : L'inflammation est un processus très complexe caractérisé par la réaction des vaisseaux sanguins aboutissants à l'accumulation de liquide et de globules blancs dans les tissus extravasculaires (Cotran, Kumar, Collins, 2000). L'inflammation résulte toujours d'une provocation de l'organisme par une cause irritante contre laquelle les tissus cherchent à se défendre, à se protéger. Symptômes de l'inflammation

- Ils sont connus depuis l'antiquité et s'extériorise par de la chaleur, de la tuméfaction, de la douleur et de la rougeur de la région ou de l'organe malade.
- A l'inverse des processus régressifs, les processus progressifs sont actifs et caractérisés par l'augmentation de processus vitaux des cellules ou des tissus.

Nécrose : La nécrose est l'arrêt pathologique et définitif des processus vitaux dans une cellule, un groupe cellulaire ou un tissu, au milieu des autres éléments restés vivants et transformations anatomiques qui en résultent (Garnier, 2000).

Macroscopiquement: Les parties atteintes se dessèchent, se raccourcissent mais conservent leur forme. Elles changent de couleur, la peau devenant noirâtre, le tissu sous cutané jaunâtre et les nerfs violacés.

Ulcères : Un ulcère est une perte de substances ou une excavation locale à la surface d'un organe ou d'un tissu produite par la détersion d'un tissu nécrosé inflammatoire. L'ulcération se rencontre le plus souvent dans les nécroses inflammatoires de la muqueuse buccale, gastrique, intestinale ou génito-urinaire et dans les inflammations sous-cutanées des membres inférieurs chez les sujets s âgés qui ont de troubles de la circulation prédisposant à une nécrose extensive (Cotran, Kumar, Collins, 2000).

Analyses statistiques: L'ensemble des résultats obtenus ont été analysés statistiquement à l'aide du test khi-carré (Schwartz, 1988). Les variables ci-dessous ont aussi fait l'objet de notre travail :-la moyenne arithmétique, -la fréquence absolue et la fréquence relative, -la fréquence sur le nombre total de sujet s.

\section{RÉSULTATS}

Les résultats sont présentés sous forme des tableaux.

Tableau 1 : Catégorie et origine des poissons

\begin{tabular}{l|l|l}
\hline Catégories & Nombre & \multicolumn{1}{|c}{ Origine } \\
\hline Tilapia & 60 & Kapolowe, Lualaba, Et angs de Lubumbashi \\
Clarias & 40 & Kapolowe, Lualaba. \\
\hline
\end{tabular}

Tableau 2 : Comparaison des fréquences des processus altératifs, inflammatoires, des troubles circulatoires et de la croissance cellulaire par catégorie des poissons

\begin{tabular}{l|c|c|c|c|c|c}
\hline & \multicolumn{6}{c}{ Catégorie des poissons et fréquence } \\
\cline { 2 - 8 } & \multicolumn{5}{|c|}{ Tilapia spp: } & \multicolumn{1}{c}{ Clarias spp: } \\
& FA & FR & FNTS & FA & FR & FNTS \\
\hline 1. Nécrose de la peau et de la nageoire & 6 & 28,57 & 10 & 14 & 35,90 & 34 \\
2. Nécrose hépatique & 10 & 47,61 & 46,66 & 13 & 33,33 & 32,50 \\
3. Nécrose du tissu musculaire et lou présence & 5 & 23,80 & 8,33 & 12 & 30,77 & 30 \\
d'ascaris adultes & & & & & & \\
TOTAL & 21 & $100 \%$ & & 39 & $100 \%$ & \\
\hline
\end{tabular}


Ipungu et al. J. Appl. Biosci. Étude des lésions anatomo-pathologiques des Tilapia et Clarias vendus sur les marchés de Lubumbashi, République Démocratique du Congo

\begin{tabular}{|c|c|c|c|c|c|c|}
\hline \multirow{3}{*}{$\begin{array}{l}\text { Processus inflammatoires et troubles } \\
\text { circulatoires }\end{array}$} & \multicolumn{6}{|c|}{ Catégorie des poissons et fréquence } \\
\hline & \multicolumn{3}{|c|}{ Tilapia spp: } & \multicolumn{3}{|c|}{ Clarias spp: } \\
\hline & FA & FR & FNTS & FA & FR & FNTS \\
\hline 1. Catarrhe intestinal hémorragique & 3 & 4,47 & 5 & 4 & 7,54 & 10 \\
\hline 2. Cystite natatoire hémorragique & 2 & 2,98 & 3,33 & 4 & 7,54 & 10 \\
\hline 3. Hémorragie branchiale & 12 & 17,91 & 20 & 10 & 18,86 & 25 \\
\hline 4. Hémorragie oculaire & 7 & 10,44 & 11,66 & 8 & 15,09 & 20 \\
\hline 5. Hémorragie sous cutanée & 14 & 20,89 & 23,33 & 6 & 11,32 & 15 \\
\hline 6. Hémorragie du tissu musculaire & 12 & 17,91 & 20 & 5 & 9,43 & 12,50 \\
\hline 7. Anémie de muqueuses buccale et oculaire & 4 & 5,97 & 7,46 & 3 & 5,66 & 7,50 \\
\hline 8. Congestion du coeur & 5 & 7,46 & 8,33 & 2 & 3,77 & 5 \\
\hline 9. 9.Ulcérations de la peau & 2 & 2,98 & 2,33 & 2 & 3,77 & 5 \\
\hline 10. Hydrothorax & 1 & 1,49 & 1,66 & 2 & 3,77 & 5 \\
\hline 11. Endophtalmie & 5 & 7,46 & 8,33 & 7 & 13,20 & 17,50 \\
\hline TOTAL & 67 & $100 \%$ & & 53 & $100 \%$ & \\
\hline \multicolumn{7}{|l|}{ Troubles de croissance cellulaire } \\
\hline \multirow[t]{3}{*}{ Troubles de la croissance cellulaire } & \multicolumn{6}{|c|}{ Catégorie des poissons et fréquence } \\
\hline & \multicolumn{3}{|c|}{ Tilapia spp: } & \multicolumn{3}{|c|}{ Clarias spp: } \\
\hline & FA & FR & FNTS & FA & FR & FNTS \\
\hline Exophtalmie & 2 & 15,38 & 3,33 & 4 & 33,33 & 10 \\
\hline Hypertrophie cardiaque & 7 & 53,84 & 11,66 & 3 & 25,00 & 7,05 \\
\hline Splénomégalie & 1 & 7,69 & 1,66 & 2 & 16,66 & 5 \\
\hline Enophtalmie & 3 & 23,07 & 5 & 3 & 25,00 & 7,50 \\
\hline TOTAL & 13 & $100 \%$ & & 12 & $100 \%$ & \\
\hline
\end{tabular}

$F a=$ Fréquence absolue (nombre des cas observés), $F R=$ Fréquence Relative = Fréquence sur le nombre total des sujets., $F N T S=$ Fréquence sur le nombre total des sujet $s$

De l'analyse du tableau ci-dessus, il ressort que les processus altératifs chez les Clarias spp représentent 42,26. p.100 des affections diagnostiquées chez cette catégorie; tandis que chez Tilapia spp, ils représentent 23,95.p. 100. De même, les troubles circulatoires représentent 57,29.p 100 chez Tilapia spp et 37,55.p.100 chez Clarias spp suivis des processus inflammatoires qui représentent 52,20.p.100 chez Clarias spp et les troubles de la croissance cellulaire représentent 12,5.p.100 chez Tilapia spp, tandis que, ils représentent 10,30.p.100 chez Clarias spp.

Tableau 3 : Fréquence des processus altératifs

\begin{tabular}{l|c|c|c}
\hline Catégories des poissons & + & - & Total \\
\hline Thlapia & 23 & 73 & 96 \\
Clarias & 41 & 56 & 97 \\
\hline
\end{tabular}

$+:$ cas positifs, $-:$ cas négatifs

L'analyse de ce tableau montre que les proportions des processus altératifs chez les Tilapia spp $(p=0,22 ; n=96)$ sont presque la moitié des celles de Clarias $\operatorname{spp}(p=0,42$; $n=97)$. La différence observée entre les fréquences relatives des Tilapia spp $(p=0,57 ; n=96)$ et Clarias spp $(p=0,37$ et $n=97)$ montre qu'elle est statistiquement significative (Chi-carré $\chi^{2}=7,27 ; \mathrm{ddl}=1$; alpha<0,50 $\left(\chi^{2}\right.$ théorique $=3,84)$. Par conséquent, les 2 espèces de poissons n'ont pas la chance de présenter les mêmes types de processus altératifs 
Ipungu et al. J. Appl. Biosci. Étude des lésions anatomo-pathologiques des Tilapia et Clarias vendus sur les marchés de Lubumbashi, République Démocratique du Congo

Tableau 4: Fréquence des troubles circulatoire

\begin{tabular}{l|c|c|c}
\hline Catégories des poissons & + & - & Total \\
\hline Tilapia & 55 & 41 & 96 \\
Clarias & 36 & 61 & 97 \\
\hline
\end{tabular}

+ : cas positifs, - : cas négatifs

Le tableau ci-dessus montre qu'il existe une différence non négligeable entre les fréquences absolues observées chez Tilapia spp (55) et Clarias spp (36). La différence observée entre les fréquences relatives des Tilapia $(p=0,57 ; n=96)$ et Clarias ( $p=0,37 ; n=97)$, montre qu'elle est statistiquement significative (Chi-carré $=7,27 ; \mathrm{ddl}=1$; alpha $<0,50(3,84)$. De ce fait comme dans le cas du processus altératif, les deux espèces n'ont pas la même chance de présenter les troubles circulatoires.

Tableau 5 : Fréquence des processus inflammatoires.

\begin{tabular}{l|c|c|c}
\hline Catégories des poissons & + & - & Total \\
\hline Thlapia & 5 & 91 & 96 \\
Clarias & 8 & 89 & 97 \\
\multicolumn{2}{c|}{ Chi-carré=0,69 ; ddl=1, alpha>0,05 ( $\chi$ théorique=3,84) } \\
\hline
\end{tabular}

$+:$ cas positifs, - : cas négatifs

La fréquence absolue des processus inflammatoires observés chez les Clarias spp (8) est légèrement supérieure à celle de Tilapia spp (5). Cependant la différence des fréquences observées entre les deux catégories des poissons Tilapia spp ( $p>0,05 ; n=96$ et
Clarias $\operatorname{spp}(p=0,08 ; n=97)$ n'est pas statistiquement significative (Chi-carré $=0,69$; ddl=1; alpha supérieur à $0,05\left(\chi^{2}\right.$ théorique $\left.=3,84\right)$. Par conséquent, les 2 espèces des poissons ne montrent pas de différences notables dans la présentation des processus inflammatoires.

Tableau 6 : Fréquence des troubles de la croissance cellulaire

\begin{tabular}{|c|c|c|c|}
\hline Catégories des poissons & + & - & Total \\
\hline THlapia & 12 & 84 & 96 \\
\hline Clarias & 10 & 87 & 97 \\
\hline
\end{tabular}

$+:$ cas positifs, - : cas négatifs

La différence observée entre les fréquences relatives de Tilapia $(p=0,57, n=96)$ et Clarias $(p=0,37, n=97)$. De l'analyse de ce tableau, il ressort que la fréquence absolue des troubles de la croissance cellulaire observée chez les Tilapia spp (12) est légèrement supérieure à celle de Clarias spp (10). Mais la différence des fréquences relatives de ces troubles de la croissance cellulaire chez les Tilapias $\operatorname{spp}(p=0,12 ; n=96)$ et chez les Clarias spp $p=0,10 ; n=97$ ) n'est pas statistiquement significative Chi-carré $=0,21 ; d d l=1$; alpha $>0,05\left(\chi^{2}\right.$ théorique=3,84). De ce fait, on peut affirmer que les 2 catégories des poissons ont la même chance de présenter ces troubles de la croissance cellulaire.

Tableau 7 : Distribution de la fréquence des processus altératifs et inflammatoires, des troubles circulatoires et de la croissance cellulaire chez Tilapia spp.

\begin{tabular}{c|c|c|c|c|c|c|c}
\hline $\mathbf{N}^{\circ}$ & Classe & Fréquence, $\mathbf{N i}$ & Centre de classe $\mathbf{x i}$ & $\mathbf{N i x i}$ & (nixi) $^{2}$ & $\mathbf{X}$ & $\mathbf{C V}$ \\
\hline 1 & $0-5$ & 28 & 2,5 & 70 & 4,900 & 290 & 47,24 \\
2 & $5-7$ & 20 & 6 & 120 & 14,400 & 6,5 & 7,69 \\
3 & $7-14$ & 48 & 10,5 & 504 & 254,016 & 12,66 & 7,34 \\
\hline
\end{tabular}

+ : cas positifs, - : cas négatifs, $X$ : moyenne arithmétique, CV : coefficient de variation 


\section{Ipungu et al. J. Appl. Biosci. Étude des lésions anatomo-pathologiques des Tilapia et Clarias vendus sur les}

marchés de Lubumbashi, République Démocratique du Congo

Ce tableau, issu également du tableau $n^{\circ} 2$, montre que toutes les valeurs de coefficients de variation sont inférieures à 50.p. 100, c'est-à dire que les valeurs ont tendance à se rapprocher de la moyenne arithmétique.
Même pris isolement, les processus altératifs et inflammatoires, troubles circulatoires et de la croissance cellulaire ont une incidence considérable et entraine une grande morbidité chez Tilapia spp.

Tableau 8 : Distribution de la fréquence des processus altératifs et inflammatoires, des troubles circulatoires et de la croissance cellulaire chez Clarias spp.

\begin{tabular}{l|c|c|c|c|c|c}
\hline $\mathbf{N}^{\circ}$ & Classe & Fréquence, Ni & Centre de classe $\mathbf{x i}$ & Nixi & $\mathbf{X}$ & $\mathbf{C V}$ \\
\hline 1 & $0-5$ & 34 & 2,5 & 85 & 3 & 33,33 \\
2 & $5-10$ & 24 & 7,5 & 180 & 8 & 117,25 \\
3 & $7-14$ & 39 & 12 & 487,5 & 12,33 & 10,05 \\
\hline
\end{tabular}

+ : cas positifs, - : cas négatifs

Ce tableau issu également du tableau $n^{\circ} 2$ permet de comparer la dispersion de processus altératifs et inflammatoires des troubles circulatoires et de la croissance cellulaire de différentes classes de la moyenne arithmétique. On constate que les affections des classes 1 et 3 , même si elles sont considérées

\section{DISCUSSION DES RESULTATS}

Lorsqu'on considère les différentes lésions anatomopathologiques diagnostiquées chez Tilapia spp et Clarias $s p p$, il ressort que les troubles circulatoires représentent $47,15 \%$ (tableau 3). Si l'on tient compte de la catégorie des poissons, on constate que Tilapia spp représente 57,29.p. 100 soit 28,49.p. 100 de l'ensemble des infections diagnostiquées comparativement à Clarias spp (37,11.p. 100 soit 18,65.p. 100 de l'ensemble des affections (tableau 3). Cette différence élevée des troubles circulatoires peut s'expliquer par le déroulement aisé du cycle évolutif du parasite et le mode d'infection facile. Plusieurs auteurs rapportent des cas de parasitoses chez les poissons d'eau douce. C'est le cas de notre recherche chez les Clarias spp. Ils affirment notamment (Giusseppe, 1953) que les ascaris et les vers semblables rencontrés chez les êtres aquatiques sont très nombreux et affectent les poissons sous forme embryonnaire, larvaire et adulte. Parmi ces trois formes, seule la forme adulte a été observée dans notre étude. Comparativement aux homéothermes, les réactions cellulaires sont moins prononcées chez les poissons. L'étendue des réactions cellulaires dépend du nombre des éléments envahisseurs de la longueur de la phase et du site de migration du parasite, comme l'a constaté aussi Hoffman (1967). Si l'infestation par les ascaris est réellement intense, on devra séquestrer les poissons et de la plus ou moins grande toxicité qui en dérive pour leurs chairs. Nul n'ignore plus aujourd'hui que les ascaris contiennent des principes hémolytiques toxiques et anémiant clairement mis en évidence par Weinberg isolement, leur incidence n'est pas à négliger car la morbidité est grande (CV< à 50.p. 100). Par contre, les affections de la classe 2(CV> 50.p. 100) n'ont pas un impact sur la santé de Clarias, que lorsqu'elles agissent en bloc. Leurs effets d'une façon séparée ont un impact moindre sur la santé de Clarias spp.

(1912), Ravenna(1916), Schart(1920) et Penso(1932) Cités Par Giusseppe (1953) et Pullin, Amon, Lazard, Legendre et Pauly (1996). Aussi l'ingestion par l'homme de poissons gravement infestés d'ascaris peut être doublement dangereuse : à la fois à cause des altérations de la chair des poissons et de l'ingestion des ascaris qui sont toujours toxiques. Anonyme (1983) affirme que les poissons provenant de Kapolowe, du fleuve Lualaba et des différents étangs piscicoles n'ont pas la même chance de présenter les mêmes aspects anatomopathologiques qui peuvent atteindre soit les organes internes, soit les organes externes. La nécrose dérmale ulcérante, la cystite natatoire, l'anémie des muqueuses buccales et oculaires observées dans cette étude corroborent cette assertion. Concernant la cystite natatoire, elle est remarquée dans plusieurs maladies des poissons. La grande participation dans la morbidité et / ou l'incidence des affections chez les poissons peut s'expliquer par l'anatomie ou la physiologie même de la peau et surtout de l'appareil respiratoire du poisson dont les branchies constituent la partie essentielle. En effet, l'épithélium branchial est assez fin pour permettre les échanges gazeux. Cette minceur la rend particulièrement vulnérable aux germes pathologiques mettant ainsi en cause la fonction respiratoire, osmorégulation et l'excrétion des déchets azotés (Anonyme, 1989). A ce sujet, Roberts (1979) précise que la maladie se manifeste tout particulièrement dans les étangs où se trouve une accumulation anormale des substances organiques. L'anémie des muqueuses buccales et 


\section{Ipungu et al. J. Appl. Biosci. Étude des lésions anatomo-pathologiques des Tilapia et Clarias vendus sur les}

marchés de Lubumbashi, République Démocratique du Congo

oculaires, quant à elle, se traduit par l'atteinte de la peau (première barrière de production contre l'environnement), les yeux, des nageoires et des branchies. Ces organes sont en fait plus sollicités parce qu'ils sont en contact permanent avec l'eau. C'est de son milieu que le poisson doit maintenir son milieu intérieur, qu'il assure les échanges indispensables à son existence. Pour ce qui est de l'inspection sanitaire, on écartera les poissons atteints d'ulcérations quelles que soient : ascite ou autres fait semblable, on se souciera d'établir, d'abord quel est l'agent étiologique qui a produit l'affection en question. Quant au trouble de la croissance cellulaire telles que exophtalmie, enophtalmie, hypertrophie du cœur, il est pratiquement aussi fréquent chez Tilapia spp (6,21.p. 100) tableau 5 que chez Clarias spp (5,18.p. 100), tandis que les processus inflammatoires observés chez les deux catégories des poissons, représentent 6,73.p. 100 (tableau 4) de l'ensemble des affections. D'après Giusseppe (1953) et De Kinkelin, Christian et Pierre (1985), les poissons atteints d'exophtalmie hémorragique

\section{CONCLUSION ET RECOMMANDATIONS}

La description des différentes lésions anatomopathologiques observées chez Tilapia spp et Clarias spp, l'évolution de leurs fréquences et la relation entre les deux catégories des poissons ont permis de tirer les conclusions suivantes:

1. Quatre groupes des lésions anatomopathologiques se dégagent, à savoir:

$$
\begin{array}{ll}
\text { - } & \text { Les processus altératifs; } \\
\text { - } & \text { Les processus inflammatoires; } \\
\text { - } & \text { Les troubles circulatoires; } \\
\hline & \text { Pables de la croissance cellulaire. }
\end{array}
$$

2. Parmi les différents aspects anatomopathologiques diagnostiqués, les troubles circulatoires représentent 47,15p. 100 de l'ensemble des affections. Quant à la distribution des fréquences, il ressort que, même pris isolement, les processus altératifs et inflammatoires; les troubles circulatoires et de croissance

\section{BIBLIOGRAPHIE}

Anonyme, 1983. La croisade de Tilapia: let ter ou noblesse de notre siècle, revue pour Quoi? Bruxelles.

Anonyme, 1989. Rapport général des travaux de la 11 ème conférence agricole régional Tenue à Lubumbashi du 21 au 17 mars 1989. Gouvernorat du Shaba, Lubumbashi.

Cotran Ramzi S , Kumar Vinay, Collins , 2000. Anatomie pathologique, troisième édition française traduite ne doivent pas être destinés à la consommation alimentaire, mais au contraire, tenus pour dangereux. Selon, Wunder (1940) cité par Giusseppe (1953), les cardiopathies chez les poissons sont considérées comme la conséquence des maladies infectieuses ou parasitaires. Le tableau 4 issu du tableau 1 montre que toutes les valeurs de coefficients de variation sont inférieures à 50 p. 100 c'est-à-dire que toutes les valeurs ont tendance à se rapprocher de la moyenne arithmétique. Même pris isolement, les processus altératifs et inflammatoires, les troubles circulatoires et de la croissance cellulaire ont une incidence considérable et une grande morbidité chez Tilapia spp. Par contre, les aspects anatomo-pathologiques de la classe 2 notamment l'hémorragie branchiale, hémorragie oculaire, hémorragie sous cutanée (CV > 50.p.100) n'ont un impact sur la santé de Clarias spp que lorsqu'elles agissent en bloc. Leurs effets d'une façon séparée ont un impact moindre sur la santé de Clarias.

cellulaire ont une incidence considérable et une grande morbidité chez Tilapia spp, tandis que les hémorragies branchiales, oculaires et sous cutanées n'ont un impact sur la santé de Clarias spp que lorsqu'elles agissent en bloc.

3. L'apparition des différents aspects anatomopathologiques n'est pas liée à la catégorie des poissons. Beaucoup de lésions anatomo-pathologiques observées chez les Tilapia spp et Clarias spp vendus dans les 3 marchés de Lubumbashi témoignent de la mauvaise qualité du produit vendu et consommé à cause du danger présenté pour l'homme (toxi-infections). Ainsi, les produits devront être retirés du commerce. De ce fait, des efforts doivent être entrepris pour séquestrer les poissons à partir de leurs lésions anatomo-pathologiques dans le cadre de la gestion de la qualité du produit de la pêche et de l'aquaculture.

de la sixième de la langue anglaise, Piccin Nuova Libraria S.p.A-Padoue, Italie.

Daube G, 2013. Gestion de la qualité du produit, D.E.S. agricultures, inédit, Liège

De Kenkelin P, Christian M et Pierre G, 1985: Précis de pathologie des poissons, édition INERA-OIE Paris

Garnier Delamare, 2000. Dictionnaire des termes de Médecine, $26{ }^{2} \mathrm{me}$ édition, Éditions Maloine, Paris. 
Giusseppe P, 1953..: Les produits de la pêche, Vigot Frères Éditeurs, Paris

Hoffman G. L, 1967. Parasites of North American fresh water fishes 486p Berkeley and Los Angeles, Univ. Calif, Presse

Kestemont P, Micha J. C Et Falter V, 1989. Les méthodes de production d'alevins de Tilapia nilotica, F.A.O, Rome.

Kestemont P, 2013.Alimentation et Nutrition des Poissons, DES-Aquaculture, CUD-Ulg-FUNDP, Namur.

Losson B, 2002: Introduction aux maladies des poissons, D.E.S aquaculture, inédit, Liège.

Mboyo 0, 1993: Amélioration de la fraicheur et de la qualité des poissons Tilapia spp vendus sur le marché local, Thèse d'agrégation en Méd.Vét, inédit, UNILU.

Philippart J .C, 2005: Les maladies des poissons transmissibles aux humains, écologie des poissons, D.E.S. inédit, Aquaculture, Liège.

Pullin R.S.V., Lazard J., Legendre M., Amon Kothias J.B. Et Pauly D., 1996. Le troisième symposium international sur le Tilapia en aquaculture, ICLARM, Philippines.

Roberts R, 1979..: Pathologie du poisson, Edition française, Malouine, Paris.

Scwart D., 1988. Méthodes de statistique à l'usage des médecins et biologistes, zème édition 11 ème tirage, Flammarion Médecine sciences, Paris 